\title{
Gravitational Waves in Newton's Gravitation and Criticism of Gravitational Waves Resulting from the General Theory of Relativity (LIGO)
}

\author{
Roman Szostek $^{1, *}$, Paweł Góralski ${ }^{2}$, Kamil Szostek ${ }^{3}$ \\ ${ }^{1}$ Rzeszow University of Technology, Department of Quantitative Methods \\ e-mail: rszostek@prz.edu.pl \\ ${ }^{2}$ Council of Gravity Research, Unit of Analysis Celestial Mechanics \\ e-mail: pg.pomylki@vp.pl \\ ${ }^{3}$ AGH University of Science and Technology, Student the Faculty EAIiIB \\ e-mail: kamil.szostek97@gmail.com \\ Corresponding: rszostek@prz.edu.pl
}

\begin{abstract}
The most important conclusion from this article is that from the General Theory of Relativity (GTR) do not result any gravitational waves, but just ordinary modulation of the gravitational field intensities caused by rotating of bodies. If the LIGO team has measured anything, it is only this modulation, rather than the gravitational wave understood as the carrier of gravity. This discussion shows that using too complicated mathematics in physics leads to erroneous interpretation of results (in this case, perhaps the tensor analysis is guilty). Formally, various things can be calculated, but without knowing what such analysis means, they can be attributed misinterpreted.

Since the modulation of gravitational field intensities has been called a gravitational wave in contemporary physics, we have also done so, although it is misleading.

In the article it was shown, that from the Newton's law of gravitation resulted an existence of gravitational waves very similar to these, which result from the General Theory of Relativity.

The article shows differences between the course of gravitational waves that result from Newton's gravitation, and the course of gravitational waves that result from the General Theory of Relativity, which measurement was announced by the LIGO (Laser Interferometer GravitationalWave Observatory) [1], [2], and [5].

According to both theories, gravitational waves are cyclical changes of the gravitational field intensities.

The article proposes a method of testing a laser interferometer for gravitational wave measurement used in the LIGO Observatory. Criticism of results published by the LIGO team was also presented.
\end{abstract}

Keywords: gravitational wave; Newton's gravitation; LIGO interferometer

\section{Introduction}

In classical mechanics, bodies that move relatively to the observer are the source of gravitational field of variable intensity. If the motion of bodies is cyclic, then they are the source of cyclic changes of the gravitational field intensities, which we will call the gravitational wave. The changes of gravitational field intensities are caused by changing distance of bodies in relation to the observer. In chapters 3 and 4 examples of gravitational waves that result from the Newton's law of gravitation are shown. 
Gravitational waves in Newton's gravitation and criticism of gravitational waves resulting from the General Theory of Relativity (LIGO) - Roman Szostek, Paweł Góralski, Kamil Szostek

In General Theory of Relativity, bodies circulating the shared centre of mass are also sources of gravitational wave, which appears as propagating vibrations of gravitational field (wrinkles of space-time) [7].

Chapter 5 presents the results announced by the Laser Interferometer Gravitational-Wave Observatory (LIGO) on the detection of gravitational wave predicted by the General Theory of Relativity.

The gravitational wave resulting from the Newton's law of gravitation has a different course than the gravitational wave registered by the LIGO team. It follows that one of these theories incorrectly describes this phenomenon, or that too many simplifications of complex model were used to study the gravitational wave within the General Theory of Relativity.

The calculations presented in this article do not take into account the time shift resulting from the finite velocity of propagation of the gravitational interactions. If the body moves away from the observer, then the time of reaching gravitational field variables to this observer becomes longer. When the body moves closer, then the time of reaching gravitational field variables is shorter. However, if the velocity of body is low in relation to the velocity of propagation of the gravitational interactions, then this effect is negligible.

In considered cases not only the gravitational field intensity changes, but also the direction of gravity effect. In the article we deal mainly with intensity. Deliberations will concern only such situations, in which the observer is in a plane of rotating bodies.

According to the Newton's law of gravitation, two homogeneous spheres of mass $m$ and $M$, located at a distance of $R$, attract each other with force

$$
F=G \frac{m M}{R^{2}}
$$

Due to Newton's second law of motion, the acceleration which a body of mass m gives to a body of mass $M$ is expressed by the following formula

$$
a=\frac{F}{M}=G \frac{m}{R^{2}}
$$

In our calculations, we assume that the gravitational constant has a value of

$$
G=6.67408(31) \cdot 10^{-11}\left[\frac{\mathrm{Nm}^{2}}{\mathrm{~kg}^{2}}\right]
$$

\section{Gravitational wave generated by a system of two rotating bodies}

We consider the two bodies circulating the shared centre of mass as shown in Figure 1. The bodies are homogeneous spheres of mass $m_{1}$ and $m_{2}$. Circles where the bodies circulate in the shared centre of mass have rays of $r_{1}$ and $r_{2}$. At any given moment the bodies are always on opposite sides of the shared centre of mass. In order for the bodies to be related by gravity in a natural way, we will assume in simulations that

$$
r_{1} m_{1}=r_{2} m_{2}=I
$$

For presented system the following applies

$$
\begin{gathered}
R^{2}=(D-x)^{2}+y^{2} \\
r_{1}^{2}=x^{2}+y^{2} \\
x=r_{1} \cos (\omega t) \\
y=r_{1} \sin (\omega t) \\
\omega=2 \pi / T
\end{gathered}
$$


Gravitational waves in Newton's gravitation and criticism of gravitational waves resulting from the General Theory of Relativity (LIGO) - Roman Szostek, Paweł Góralski, Kamil Szostek

On this basis, we obtain the following

$$
R^{2}=D^{2}+x^{2}+y^{2}-2 x D=D^{2}+r_{1}^{2}-2 D r_{1} \cos (\omega t)
$$

Based on (2), the gravity field intensity from mass $m_{l}$ has at the detector point the value of

$$
a_{1}=G \frac{m_{1}}{R^{2}} \quad\left[\frac{\mathrm{m}}{\mathrm{s}^{2}}=\frac{\mathrm{N}}{\mathrm{kg}}\right]
$$

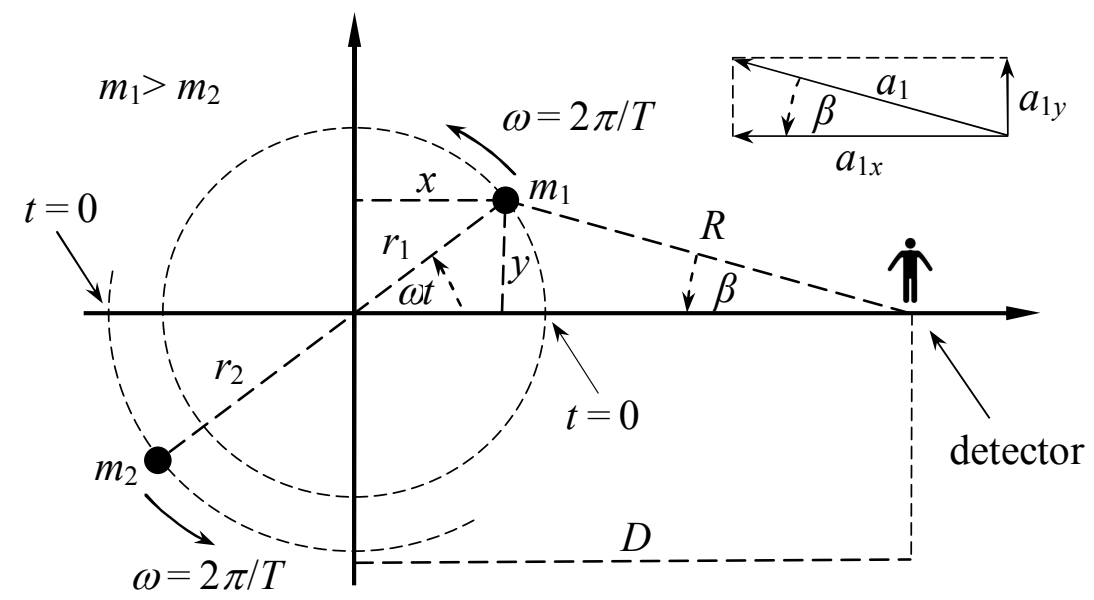

Fig. 1. Two bodies of $m_{1}$ and $m_{2}$ mass rotating in a circle of $r_{1}$ and $r_{2}$ radius.

After taking into account (10), the following results are obtained

$$
a_{1}=G \frac{m_{1}}{D^{2}+r_{1}^{2}-2 D r_{1} \cos (\omega t)}
$$
values

The components of gravitational field intensity, as shown in Figure 1, have the following

$$
\begin{gathered}
a_{1 x}=a_{1} \cos \beta=a_{1} \frac{D-x}{R} \\
a_{1 y}=a_{1} \sin \beta=a_{1} \frac{y}{R}
\end{gathered}
$$

On the basis of (7), (8), (10) and (12) we obtain the following

$$
\begin{aligned}
& a_{1 x}=G \frac{m_{1}}{D^{2}+r_{1}^{2}-2 D r \cos (\omega t)} \frac{D-r_{1} \cos (\omega t)}{\sqrt{D^{2}+r_{1}^{2}-2 D r_{1} \cos (\omega t)}} \\
& a_{1 y}=G \frac{m_{1}}{D^{2}+r_{1}^{2}-2 D r \cos (\omega t)} \frac{r_{1} \sin (\omega t)}{\sqrt{D^{2}+r_{1}^{2}-2 D r_{1} \cos (\omega t)}}
\end{aligned}
$$

On this basis, we obtain the following

$$
\begin{aligned}
& a_{1 x}=G m_{1} \frac{D-r_{1} \cos (\omega t)}{\left(D^{2}+r_{1}^{2}-2 D r_{1} \cos (\omega t)\right)^{3 / 2}} \\
& a_{1 y}=G m_{1} \frac{r_{1} \sin (\omega t)}{\left(D^{2}+r_{1}^{2}-2 D r_{1} \cos (\omega t)\right)^{3 / 2}}
\end{aligned}
$$

In case of body with $m_{2}$ mass, the above formulae differ only in an angle, which is not $\omega t$, but $\omega t+\pi$. For the body $m_{2}$, the relation (12), (17), (18) are in a form of 
Gravitational waves in Newton's gravitation and criticism of gravitational waves resulting from the General Theory of Relativity (LIGO) - Roman Szostek, Paweł Góralski, Kamil Szostek

$$
\begin{gathered}
a_{2}=G \frac{m_{2}}{D^{2}+r_{2}^{2}-2 D r_{2} \cos (\omega t+\pi)}=G \frac{m_{2}}{D^{2}+r_{2}^{2}+2 D r_{2} \cos (\omega t)} \\
a_{2 x}=G m_{2} \frac{D-r_{2} \cos (\omega t+\pi)}{\left(D^{2}+r_{2}^{2}-2 D r_{2} \cos (\omega t+\pi)\right)^{3 / 2}}=G m_{2} \frac{D+r_{2} \cos (\omega t)}{\left(D^{2}+r_{2}^{2}+2 D r_{2} \cos (\omega t)\right)^{3 / 2}} \\
a_{2 y}=G m_{2} \frac{r_{2} \sin (\omega t+\pi)}{\left(D^{2}+r_{2}^{2}-2 D r_{2} \cos (\omega t+\pi)\right)^{3 / 2}}=G m_{2} \frac{-r_{2} \sin (\omega t)}{\left(D^{2}+r_{2}^{2}+2 D r_{2} \cos (\omega t)\right)^{3 / 2}}
\end{gathered}
$$

The components of gravitational field intensity from two bodies are

$$
\begin{gathered}
a_{x}=a_{1 x}+a_{2 x}=G\left[\frac{m_{1}\left(D-r_{1} \cos (\omega t)\right)}{\left(D^{2}+r_{1}^{2}-2 D r_{1} \cos (\omega t)\right)^{3 / 2}}+\frac{m_{2}\left(D+r_{2} \cos (\omega t)\right)}{\left(D^{2}+r_{2}^{2}+2 D r_{2} \cos (\omega t)\right)^{3 / 2}}\right] \\
a_{y}=a_{1 y}+a_{2 y}=G \sin (\omega t)\left[\frac{r_{1} m_{1}}{\left(D^{2}+r_{1}^{2}-2 D r_{1} \cos (\omega t)\right)^{3 / 2}}-\frac{r_{2}}{\left(D^{2}+r_{2}^{2}+2 D r_{2} \cos (\omega t)\right)^{3 / 2}}\right]
\end{gathered}
$$

The gravitational field intensity from two bodies is

$$
a=\sqrt{a_{x}^{2}+a_{y}^{2}}
$$

In some cases we will assume that the distance of circulating bodies may change. Then their distance will decrease linearly in time, according to the relation

$$
r_{i}(t)=r_{i}(0) \cdot\left(1-\frac{t}{3 T}\right)
$$

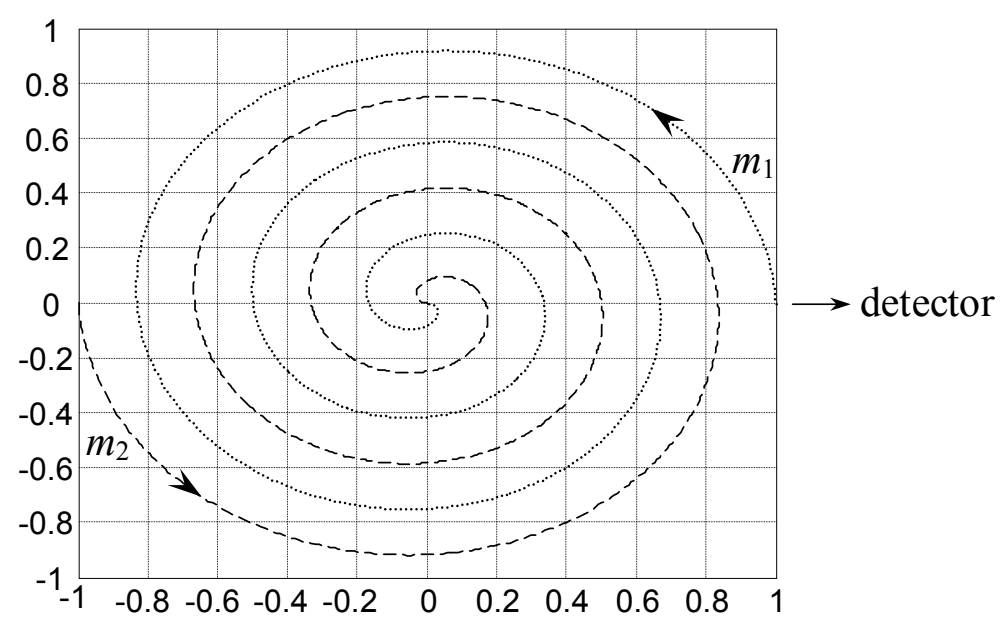

Fig. 2. Trajectory of body movement assumed in some simulations, for $r_{1}(0)=r_{2}(0)=1 \mathrm{~m}$.

In this case, the bodies are not naturally related by gravity and their movement is artificially forced. In this case, the bodies fall over each other in a spiral during $3 T$ (Figure 2).

\subsection{Two bodies of equal mass}

We assume that two rotating bodies have identical mass $m_{1}=m_{2}=m$.

Figure 3 shows the gravitational field intensity (24) in a distance of $D=1.5 \mathrm{~m}$ from rotating bodies. As can be seen from the figure for $D \approx r$, the variations in intensity are not sinusoidal. 
Gravitational waves in Newton's gravitation and criticism of gravitational waves resulting from the General Theory of Relativity (LIGO) - Roman Szostek, Paweł Góralski, Kamil Szostek

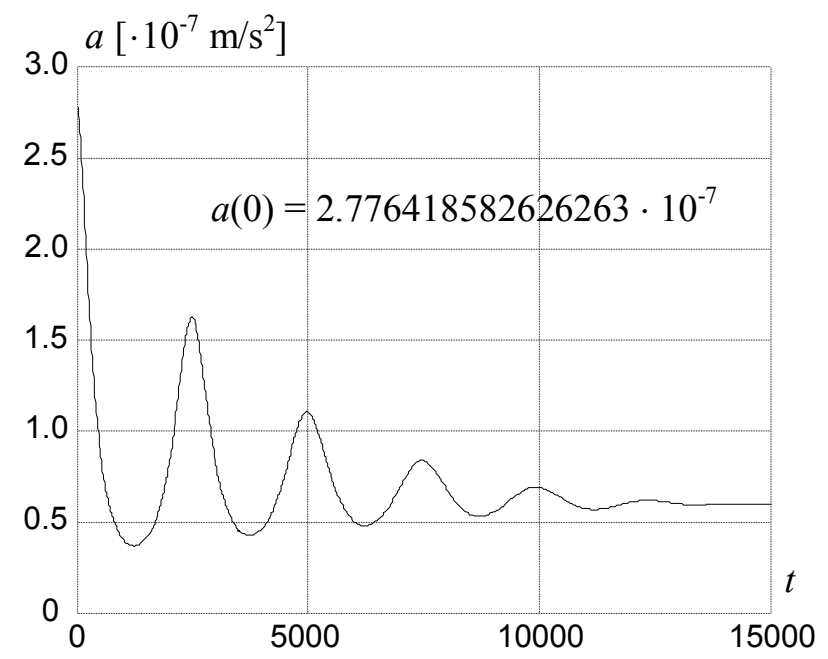

Fig. 3. The gravitational field intensity for $D=1.5 \mathrm{~m}, r(0)=1 \mathrm{~m}, m_{1}=m_{2}=1000 \mathrm{~kg}, T=5000$.

Figure 4 shows the gravitational field intensity (24) in a distance of $D=100000 \mathrm{~m}$, which bodies are a source with mass of $m=10000 \mathrm{~kg}$. For $D \gg r$ the intensity changes are similar to sinusoidal.

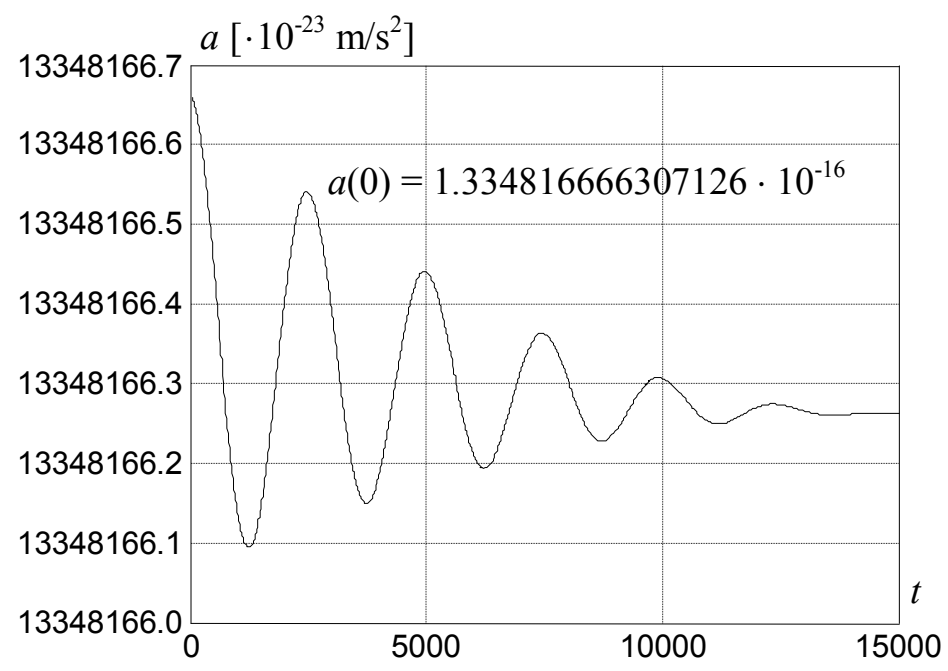

Fig. 4. The gravitational field intensity for $D=100000 \mathrm{~m}, r(0)=10 \mathrm{~m}, m_{1}=m_{2}=10000 \mathrm{~kg}, T=5000$.

The presented analysis shows that when two bodies rotate around each other, they are a source of gravitational field of variable intensity. If the bodies get closer to each other by the spiral, then the amplitude of changes decreases. Local maxima occur twice in one full body rotation.

\subsection{Two bodies of different mass}

We assume that two rotating bodies have different mass $m_{1}>m_{2}$. Figure 5 shows the gravitational field intensity (24) in a distance of $D=20 \mathrm{~m}$ from, which bodies are a source with mass of $m_{1}=350 \mathrm{~kg}$ and $m_{2}=250 \mathrm{~kg}$. This time the distance of rotating bodies is constant, i.e. $r_{1}(t)=$ constans $=10 \mathrm{~m}$. After taking into account (4) we receive $r_{2}(t)=$ constans $=14 \mathrm{~m}$.

Figure 5 shows two local maxima at full body rotation. The smaller one occurs when a body of higher mass is closer to the detector than a body of lower mass. The larger one occurs when a body of lower mass is closer to the detector than a body of higher mass. This is because the smaller body circulates around a larger orbit, so it comes closer to the detector (formula (4) applies). 
Gravitational waves in Newton's gravitation and criticism of gravitational waves resulting from the General Theory of Relativity (LIGO) - Roman Szostek, Paweł Góralski, Kamil Szostek

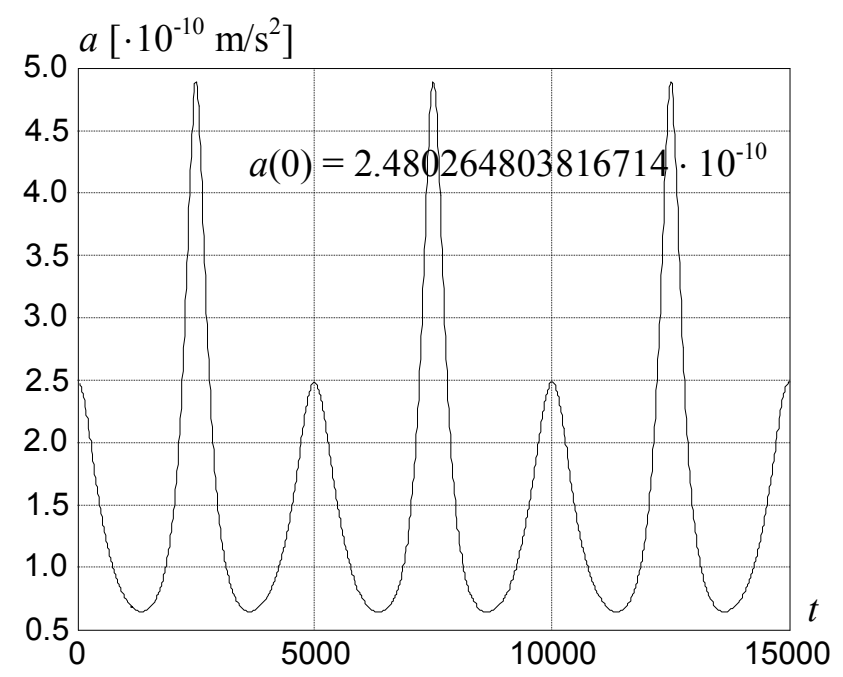

Fig. 5. The gravitational field intensity for

$D=20 \mathrm{~m}, r_{1}(t)=$ constans $=10 \mathrm{~m}, r_{2}(t)=$ constans $=14 \mathrm{~m}, m_{1}=350 \mathrm{~kg}, m_{2}=250 \mathrm{~kg}, T=5000$.

When the detector is more distant from the rotating bodies, then the difference between maxima disappears. For example, for the same system of bodies as in Figure 5, the gravitational field intensity seen from a distance of $D=450 \mathrm{~m}$ has two, almost identical, maxima at full rotation of bodies (Figure 6).

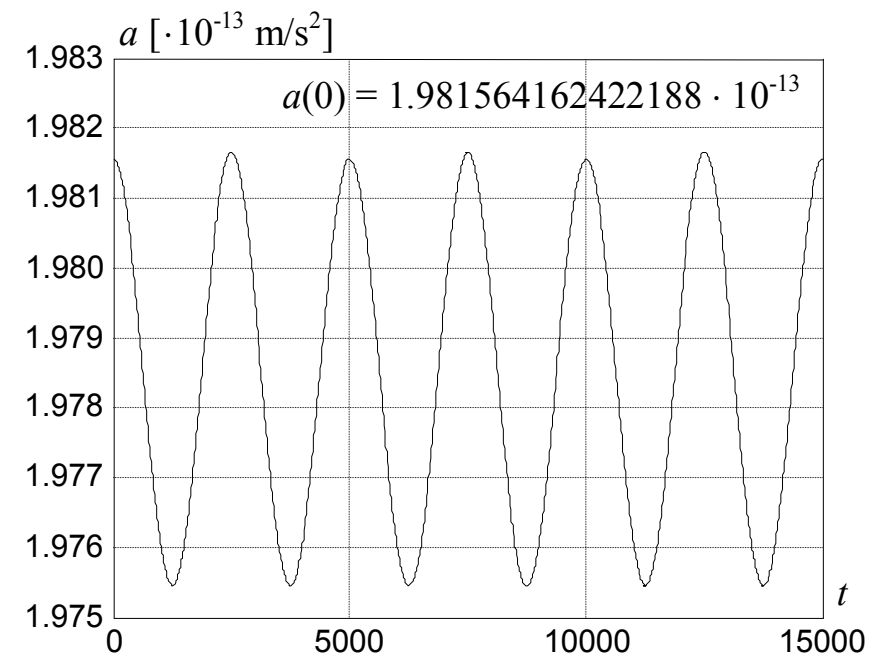

Fig. 6. The gravitational field intensity for

$D=450 \mathrm{~m}, r_{1}(t)=$ constans $=10 \mathrm{~m}, r_{2}(t)=$ constans $=14 \mathrm{~m}, m_{1}=350 \mathrm{~kg}, m_{2}=250 \mathrm{~kg}, T=5000$.

\subsection{Gravitational wave caused by four moons of Jupiter}

In order to determine the gravitational field intensity from the four Galileo moons of Jupiter, we have carried out a numerical simulation (for the data from Table 1). On its basis, the intensity of gravitational field seen from Earth was determined, the source of which are four Galileo moons of Jupiter.

For reasons of simplification, it has been assumed that the orbits of these four moons are in the same plane as the Earth. It was also assumed that at initial moment $t=0$, the Jupiter and all moons of Jupiter are in conjunction with the Earth, i.e. lie on a section connecting the centre of Earth with the centre of Jupiter. Since the mass of Jupiter is much greater than the mass of moons, for simplicity it was assumed that Jupiter is motionless in relation to the Earth. For this reason, Jupiter does not influence the gravitational field intensity changes from his moons and has not been taken into account in simulation. 
Gravitational waves in Newton's gravitation and criticism of gravitational waves resulting from the General Theory of Relativity (LIGO) - Roman Szostek, Paweł Góralski, Kamil Szostek

\begin{tabular}{|l|l|}
\hline Distance of Jupiter from the Earth & $D=6,3 \cdot 10^{11} \mathrm{~m}$ \\
\hline Mass Io & $m_{I}=8,9 \cdot 10^{22} \mathrm{~kg}$ \\
\hline Orbit radius Io & $r_{I}=4,2 \cdot 10^{8} \mathrm{~m}$ \\
\hline Rotation velocity Io & $\omega_{I}=2 \pi / T_{I}=2 \pi /\left(1,5 \cdot 10^{5} \mathrm{~s}\right)=2,1 \cdot 10^{-5} 1 / \mathrm{s}$ \\
\hline Europe mass & $m_{E}=4,8 \cdot 10^{22} \mathrm{~kg}$ \\
\hline Orbit radius of Europe & $r_{E}=6,7 \cdot 10^{8} \mathrm{~m}$ \\
\hline Rotation velocity of Europe & $\omega_{E}=2 \pi / T_{E}=2 \pi /\left(3,1 \cdot 10^{5} \mathrm{~s}\right)=2,0 \cdot 10^{-5} 1 / \mathrm{s}$ \\
\hline Ganymede mass & $m_{G}=1,5 \cdot 10^{23} \mathrm{~kg}$ \\
\hline Orbit radius of Ganymede & $r_{G}=1,1 \cdot 10^{9} \mathrm{~m}$ \\
\hline Rotation velocity of Ganymede & $\omega_{G}=2 \pi / T_{G}=2 \pi /\left(6,2 \cdot 10^{5} \mathrm{~s}\right)=1,0 \cdot 10^{-5} 1 / \mathrm{s}$ \\
\hline Callista mass & $m_{K}=1,1 \cdot 10^{23} \mathrm{~kg}$ \\
\hline Orbit radius of Callista & $r_{K}=1,9 \cdot 10^{9} \mathrm{~m}$ \\
\hline Rotation velocity of Callista & $\omega_{K}=2 \pi / T_{K}=2 \pi /\left(1,4 \cdot 10^{6} \mathrm{~s}\right)=4,4 \cdot 10^{-6} 1 / \mathrm{s}$ \\
\hline
\end{tabular}

Table 1. Sizes accepted in calculations for Jupiter and its four moons.

Results of simulation were presented in Figure 7.

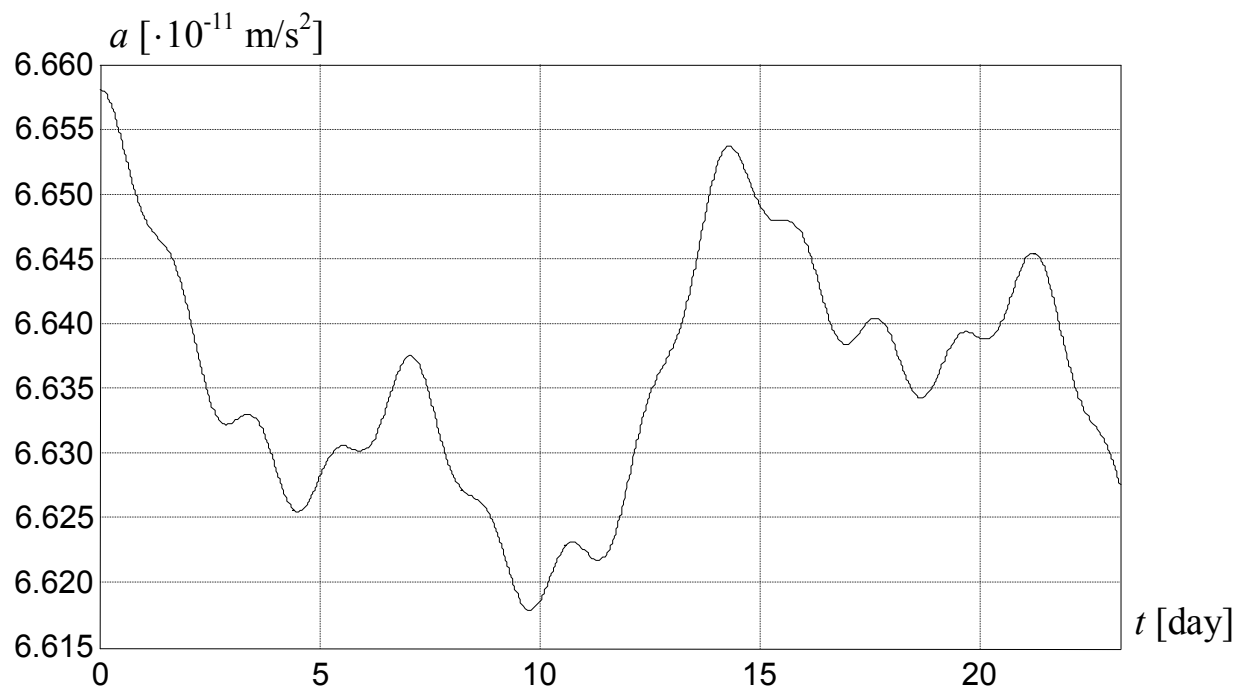

Fig. 7. The gravitational field intensity of four Jupiter moons, seen from the Earth (Table 1). The gravitational field intensity from Jupiter is not taken into account.

\section{Gravitational wave as seen by the rotating observer}

We consider the rotating observer as shown in Figure 8 . The observer is gravitated by a homogeneous sphere with a mass of $m$. For presented system, the following occurs

$$
\begin{gathered}
R^{2}=(d-x)^{2}+y^{2} \\
r^{2}=x^{2}+y^{2} \\
x=r \cos (\omega t) \\
y=r \sin (\omega t) \\
\omega=2 \pi / T
\end{gathered}
$$

On this basis, we obtain the following

$$
R^{2}=D^{2}+x^{2}+y^{2}-2 x D=D^{2}+r^{2}-2 D r \cos (\omega t)
$$


Gravitational waves in Newton's gravitation and criticism of gravitational waves resulting from the General Theory of Relativity (LIGO) - Roman Szostek, Paweł Góralski, Kamil Szostek

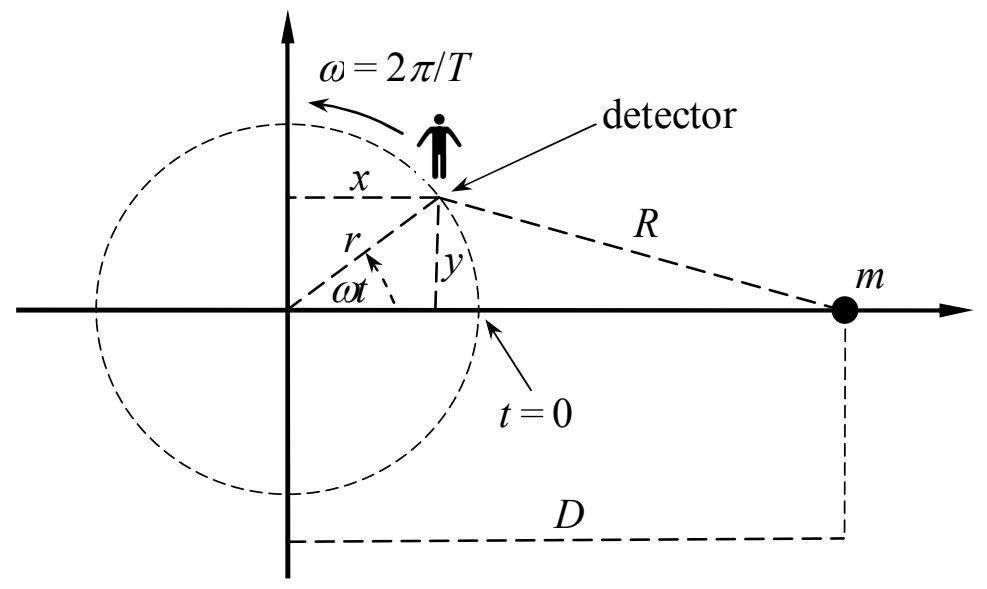

Fig. 8. Rotating observer in a circle with a radius of $r$. i.e.

After placing to (11) we obtain a gravitational field intensity dependency identical to (12),

$$
a=G \frac{m}{D^{2}+r^{2}-2 D r \cos (\omega t)}
$$

The case in which the observer rotates is qualitatively different from the case shown in Chapter 2, where the two bodies rotate around each other. In this case, the gravitational wave does not propagate in a space where the source of gravity is motionless. Changes of the gravitational field intensity occur only for a moving observer. But formula (32) will also apply in a symmetrical situation when the observer is motionless and the source of wave circulates along a suitable trajectory. Formula (32) will also apply if the body and observer change places.

\subsection{Gravitational wave from the Moon}

The situation shown in Figure 8 may be a model of the Earth-Moon system. The observer is located on the surface of Earth in a plane of the Moon orbit. The source of gravity is the Moon. In the Moon system, the observer rotates around the center of $t$ Earth. We assume the values shown in Table 2. The observer's rotation period is slightly longer than 24 hours due to the Moon's movement around the Earth.

\begin{tabular}{|l|l|}
\hline Average radius of Earth & $r=6371,0 \mathrm{~km}=6,4 \cdot 10^{6} \mathrm{~m}$ \\
\hline Average radius of Moon orbit & $D=384404 \mathrm{~km}=3,8 \cdot 10^{8} \mathrm{~m}$ \\
\hline Moon mass & $m=7,3 \cdot 10^{22} \mathrm{~kg}$ \\
\hline Rotation velocity of the observer & $\omega=2 \pi / T=2 \pi /(24,84 \mathrm{~h})=2 \pi /(89428 \mathrm{~s})=7,02 \cdot 10^{-5} 1 / \mathrm{s}$ \\
\hline
\end{tabular}

Table 2. Sizes accepted in calculations for the Moon.

The diagram of gravitational wave from the Moon calculated on the basis of (32) is shown in Figure 9. This gravitational wave is the cause of tides and outflows.

The gravitational wave amplitude of the Moon is

$$
\frac{a_{K_{-} \max }-a_{K_{-} \min }}{2}=\frac{a_{K}(0)-a_{K}(T / 2)}{2}=\frac{2.2743 \cdot 10^{-6}}{2} \mathrm{~m} / \mathrm{s}^{2}=1.1372 \cdot 10^{-6} \mathrm{~m} / \mathrm{s}^{2}
$$


Gravitational waves in Newton's gravitation and criticism of gravitational waves resulting from the General Theory of Relativity (LIGO) - Roman Szostek, Paweł Góralski, Kamil Szostek

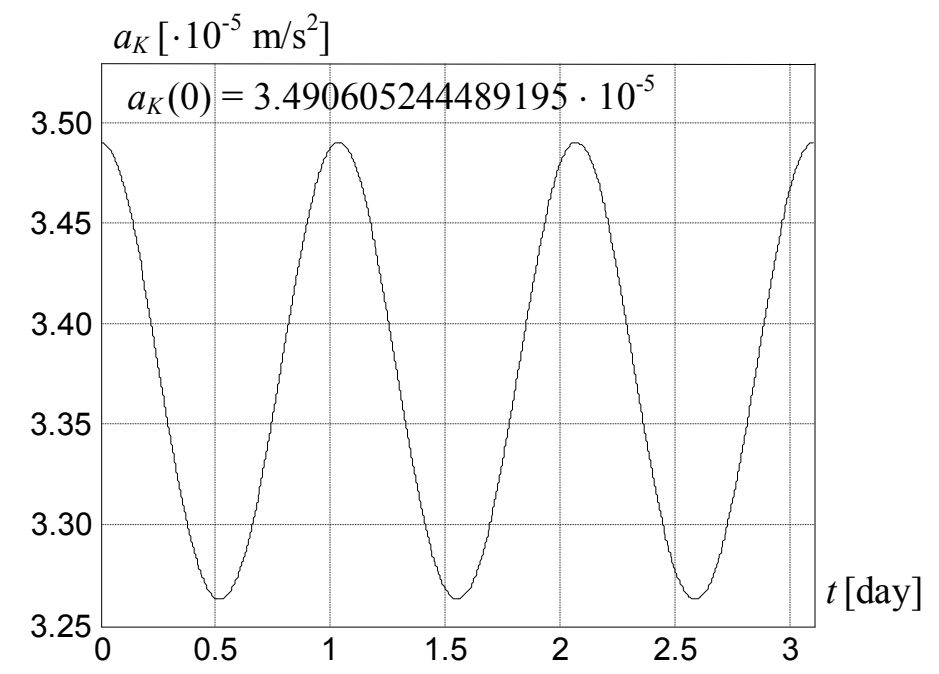

Fig. 9. The gravitational field intensity from the Moon (Table 2).

\subsection{Gravitational wave from the Sun}

The situation shown in Figure 8 may also be a model of the Earth-Sun system. The observer is located on the surface of Earth. The source of gravity is the Sun. In the solar system, the observer rotates around the centre of Earth. We assume the values shown in Table 3.

\begin{tabular}{|l|l|}
\hline Average radius of Earth & $r=6371,0 \mathrm{~km}=6,4 \cdot 10^{6} \mathrm{~m}$ \\
\hline Average radius of Earth orbit & $D=149,6 \cdot 10^{6} \mathrm{~km}=1,5 \cdot 10^{11} \mathrm{~m}$ \\
\hline Sun mass & $m=2,0 \cdot 10^{30} \mathrm{~kg}$ \\
\hline Rotation velocity of the observer & $\omega=2 \pi / T=2 \pi /(24 \mathrm{~h})=2 \pi /(86400 \mathrm{~s})=7,27 \cdot 10^{-5} 1 / \mathrm{s}$ \\
\hline
\end{tabular}

Table 3. Sizes accepted in calculations for the Sun.

The diagram of gravitational wave from the Sun calculated on the basis of (32) is shown in Figure 10 .

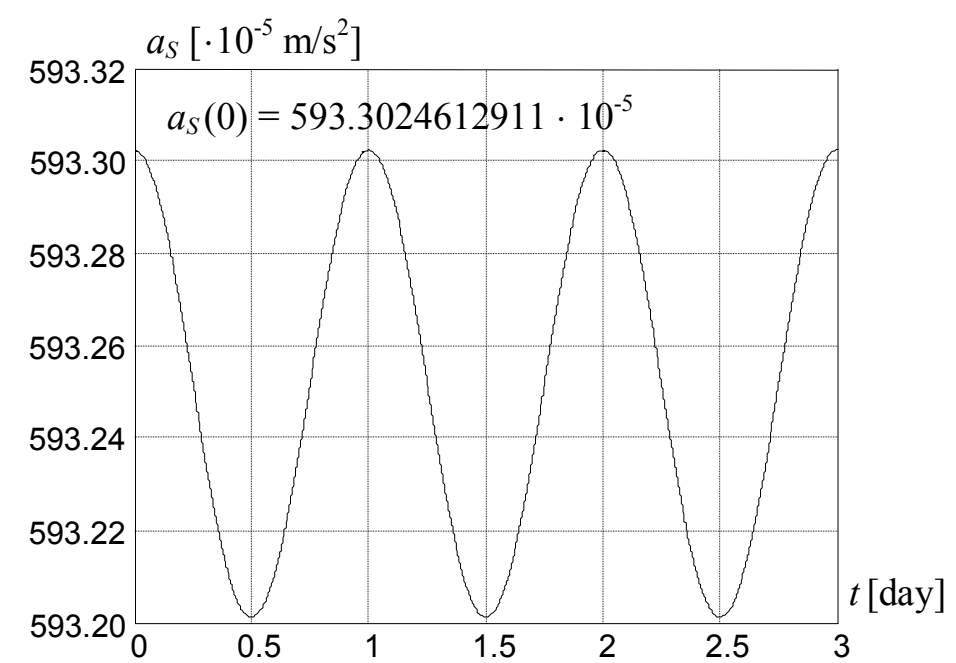

Fig. 10. The gravitational field intensity from the Sun (Table 3).

The amplitude of the gravitational wave from the Sun is

$$
\frac{a_{S_{-} \max }-a_{S_{-} \min }}{2}=\frac{a_{S}(0)-a_{S}(T / 2)}{2}=\frac{1.0125 \cdot 10^{-6}}{2} \mathrm{~m} / \mathrm{s}^{2}=0.5063 \cdot 10^{-6} \mathrm{~m} / \mathrm{s}^{2}
$$


Gravitational waves in Newton's gravitation and criticism of gravitational waves resulting from the General Theory of Relativity (LIGO) - Roman Szostek, Paweł Góralski, Kamil Szostek

The gravitational waves from the Moon and Sun cause tides on the Earth. This means that the phenomenon of gravitational waves is known and observed on the Earth from always. From these observations it appears that the gravitational wave deforms the shape of bodies.

The intensity of the gravitational field $a_{K}$ from the Moon (Figure 9) is several hundred times smaller than the intensity of gravitational field $a_{S}$ originating from the Sun (Figure 10). However, the Moon is much closer to Earth, and therefore the maximum change of gravitational field intensity from the Moon (33) is 2.25 times greater than the maximum change of gravitational field intensity from the Sun (34). For this reason, the sea tides and outflows caused by the Moon are greater than those caused by the Sun.

\section{Absolute changes of the gravitational field intensity (amplitude)}

According to the General Theory of Relativity mass influences the geometry of space-time. According to the creators of LIGO detector, this decoder can measure relative changes in the length of two arms of this interferometer [1]. On this basis, changes in gravitational field intensity can be measured indirectly. The LIGO Interferometer cannot measure the absolute value of gravitational field intensity. Its task is to indirectly measure changes in gravitational field intensity.

Such a measurement is not affected by how large the gravitational field is, but by the changes in its value (absolute changes). Therefore, in the following subsections we are going to determine absolute changes of the gravitational field intensity for two previously considered cases.

\subsection{Gravitational wave amplitude caused by two rotating bodies}

Now we will determine the absolute changes of gravity field intensity for the case discussed in Chapter 2, two bodies related by gravity.

\subsubsection{Component $y$ of the gravitational wave}

First we will determine the component changes $y$. On the basis of (23), taking into account condition (4), the following are obtained

$$
\begin{gathered}
a_{y}=G \frac{1}{D^{3}} \sin (\omega t)\left[\frac{r_{1} m_{1}}{\left(1+\frac{r_{1}^{2}}{D^{2}}-2 \frac{r_{1}}{D} \cos (\omega t)\right)^{3 / 2}}-\frac{r_{1} m_{1}}{\left(1+\frac{r_{2}^{2}}{D^{2}}+2 \frac{r_{2}}{D} \cos (\omega t)\right)^{3 / 2}}\right] \\
D>\left(r_{1}+r_{2}\right) \Rightarrow a_{y} \approx G \frac{r_{1} m_{1}}{D^{3}} \sin (\omega t)\left[\frac{1}{\left(1-2 \frac{r_{1}}{D} \cos (\omega t)\right)^{3 / 2}}-\frac{1}{\left(1+2 \frac{r_{2}}{D} \cos (\omega t)\right)^{3 / 2}}\right]
\end{gathered}
$$

Based on developing into the Taylor series, it is possible to show that

$$
x \approx 0 \Rightarrow \frac{1}{(1-2 x)^{3 / 2}} \approx 1+3 x \wedge \frac{1}{(1+2 x)^{3 / 2}} \approx 1-3 x
$$

On this basis, formula (36), it is possible to write in approximated form

$$
\begin{gathered}
D>>\left(r_{1}+r_{2}\right) \Rightarrow a_{y} \approx G \frac{r_{1} m_{1}}{D^{3}} \sin (\omega t)\left[\left(1+3 \frac{r_{1}}{D} \cos (\omega t)\right)-\left(1-3 \frac{r_{2}}{D} \cos (\omega t)\right)\right] \\
D>>\left(r_{1}+r_{2}\right) \Rightarrow a_{y} \approx 3 G \frac{r_{1} m_{1}\left(r_{1}+r_{2}\right)}{D^{4}} \sin (\omega t) \cos (\omega t)
\end{gathered}
$$
following

On the basis of the designation adopted in (4) and the trigonometric formula we obtain the

$$
D>>\left(r_{1}+r_{2}\right) \Rightarrow a_{y} \approx \frac{3}{2} G \frac{I\left(r_{1}+r_{2}\right)}{D^{4}} \sin (2 \omega t)
$$


Gravitational waves in Newton's gravitation and criticism of gravitational waves resulting from the General Theory of Relativity (LIGO) - Roman Szostek, Paweł Góralski, Kamil Szostek

It results that the amplitude of component $\mathrm{y}$ of the gravitational field intensity is

$$
D>>\left(r_{1}+r_{2}\right) \Rightarrow A_{y} \approx \frac{3}{2} G \frac{I\left(r_{1}+r_{2}\right)}{D^{4}}
$$

\subsubsection{Component $x$ of the gravitational wave} following

Now we will determine the changes in component $x$. On the basis of (22) we obtain the

$$
\begin{gathered}
a_{x}=G \frac{1}{D^{2}}\left[m_{1} \frac{\left.1-\frac{r_{1}}{D} \cos (\omega t)\right)}{\left(1+\frac{r_{1}^{2}}{D^{2}}-2 \frac{r_{1}}{D} \cos (\omega t)\right)^{3 / 2}}+m_{2} \frac{\left.1+\frac{r_{2}}{D} \cos (\omega t)\right)}{\left(1+\frac{r_{2}^{2}}{D^{2}}+2 \frac{r_{2}}{D} \cos (\omega t)\right)^{3 / 2}}\right] \\
D>\left(r_{1}+r_{2}\right) \Rightarrow a_{x} \approx G \frac{1}{D^{2}}\left[m_{1} \frac{\left.1-\frac{r_{1}}{D} \cos (\omega t)\right)}{\left(1-2 \frac{r_{1}}{D} \cos (\omega t)\right)^{3 / 2}}+m_{2} \frac{\left.1+\frac{r_{2}}{D} \cos (\omega t)\right)}{\left(1+2 \frac{r_{2}}{D} \cos (\omega t)\right)^{3 / 2}}\right]
\end{gathered}
$$

Based on developing into the Taylor series, it is possible to show that

$$
x \approx 0 \Rightarrow \frac{1-x}{(1-2 x)^{3 / 2}} \approx 1+2 x+\frac{9}{2} x^{2} \wedge \frac{1+x}{(1+2 x)^{3 / 2}} \approx 1-2 x+\frac{9}{2} x^{2}
$$

On this basis, formula (43), it is possible to write in approximated form

$$
\begin{aligned}
D>\left(r_{1}+r_{2}\right) \Rightarrow a_{x} \approx G \frac{1}{D^{2}} & {\left[m_{1}\left[1+2 \frac{r_{1}}{D} \cos (\omega t)+\frac{9}{2} \frac{r_{1}^{2}}{D^{2}} \cos ^{2}(\omega t)\right]+\right.} \\
& \left.+m_{2}\left[1-2 \frac{r_{2}}{D} \cos (\omega t)+\frac{9}{2} \frac{r_{2}^{2}}{D^{2}} \cos ^{2}(\omega t)\right]\right]
\end{aligned}
$$

On the basis of (4) we obtain

$$
\begin{gathered}
D>\left(r_{1}+r_{2}\right) \Rightarrow a_{x} \approx G \frac{1}{D^{2}}\left[m_{1}+m_{2}+\frac{9}{2} \frac{r_{1}^{2} m_{1}}{D^{2}} \cos ^{2}(\omega t)+\frac{9}{2} \frac{r_{2}^{2} m_{2}}{D^{2}} \cos ^{2}(\omega t)\right] \\
D>\left(r_{1}+r_{2}\right) \Rightarrow a_{x} \approx G \frac{1}{D^{2}}\left[m_{1}+m_{2}+\frac{9}{2} \frac{I\left(r_{1}+r_{2}\right)}{D^{2}} \cos ^{2}(\omega t)\right]
\end{gathered}
$$

On the basis of the trigonometric formula

$$
\cos ^{2}(\omega t)=\frac{1}{2}(\cos (2 \omega t)+1)
$$

we obtain the following

$$
D>>\left(r_{1}+r_{2}\right) \Rightarrow a_{x} \approx G \frac{m_{1}+m_{2}}{D^{2}}+\frac{9}{4} G \frac{I\left(r_{1}+r_{2}\right)}{D^{4}}(\cos (2 \omega t)+1)
$$

On the basis of (4), it is possible to show that

$$
D>>\sqrt{r_{1} \cdot r_{2}} \Rightarrow \frac{m_{1}+m_{2}}{D^{2}}>>\frac{9}{4} \frac{I\left(r_{1}+r_{2}\right)}{D^{4}}
$$

I.e. the formula (49), it is possible to write in approximated form

$$
D>>\left(r_{1}+r_{2}\right) \Rightarrow a_{x} \approx G \frac{m_{1}+m_{2}}{D^{2}}+\frac{9}{4} G \frac{I\left(r_{1}+r_{2}\right)}{D^{4}} \cos (2 \omega t)
$$

Intensity $a_{x}(51)$ consists of a constant part in time with a value of 
Gravitational waves in Newton's gravitation and criticism of gravitational waves resulting from the General Theory of Relativity (LIGO) - Roman Szostek, Paweł Góralski, Kamil Szostek

$$
D>>\left(r_{1}+r_{2}\right) \Rightarrow \bar{a}_{x} \approx G \frac{1}{D^{2}}\left(m_{1}+m_{2}\right)
$$

and parts of variable in the time with value of

$$
D>>\left(r_{1}+r_{2}\right) \Rightarrow \Delta a_{x} \approx \frac{9}{4} G \frac{I\left(r_{1}+r_{2}\right)}{D^{4}} \cos (2 \omega t)
$$

It results that the amplitude of $\mathrm{x}$ component of the gravitational field intensity is

$$
D>>\left(r_{1}+r_{2}\right) \Rightarrow A_{x} \approx \frac{9}{4} G \frac{I\left(r_{1}+r_{2}\right)}{D^{4}}
$$

\subsubsection{Law for the gravitational wave amplitude}

The simplified formulae (40), (41) and (52), (53), (54) have been numerically verified and in a satisfactory way are in line with the exact formulae (23) and (22).

According to (52), when two bodies rotate, the resultant gravitational field intensity is inversely proportional to $D^{2}$ and proportional to the sum of mass.

Formulas (41) and (54) express changes in components of the gravitational field intensity (amplitudes). They show that the amplitude of gravitational wave generated by two bodies related by gravity is inversely proportional to $D^{4}$, proportional to the distance of $r_{1}+r_{2}$ between rotating bodies and proportional to the moment of inertia $I$ (4).

Figure 11 shows components of the gravitational field intensity: time constant component (52), two time variable components (40) and (53). For the observer, component y causes changes of the direction of gravitational field intensity vector. Component $x$ causes changes of the value of gravitational field intensity vector.

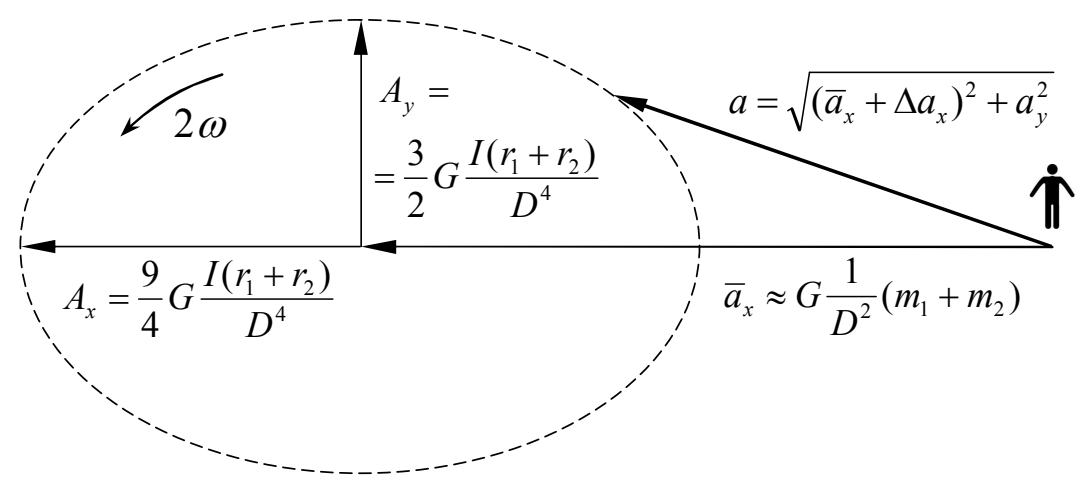

Fig. 11. Components of the gravitational field intensity caused two rotating each other bodies related by gravity, when

$$
D>r_{1}+r_{2} \text {. }
$$

Formulas (40) and (53) indicate that the frequency of gravitational wave is twice as high as the frequency with which bodies rotate.

On the basis of above deductions we can formulate the law for gravitational wave amplitude coming from two bodies related by gravity. If the parameters of two rotating each other bodies are determined with the index $\mu$ and the parameters of two other rotating each other bodies are determined with the index $\eta$, then the following occurs

$$
\frac{I_{\eta}\left(r_{\eta 1}+r_{\eta 2}\right)}{D_{\eta}^{4}} \approx \frac{I_{\mu}\left(r_{\mu 1}+r_{\mu 2}\right)}{D_{\mu}^{4}} \underset{D_{\mu} \gg r_{\mu 1}+r_{\mu 2}}{\stackrel{D_{\eta} \gg r_{\eta 1}+r_{\eta 2}}{\Rightarrow}} A_{\eta} \approx A_{\mu}
$$


Gravitational waves in Newton's gravitation and criticism of gravitational waves resulting from the General Theory of Relativity (LIGO) - Roman Szostek, Paweł Góralski, Kamil Szostek

\subsection{Gravitational wave amplitude as seen by the rotating observer} in Chapter 3.

Now we will determine the absolute changes of gravity field intensity for the case discussed

According to the formula (32) we obtain

$$
\begin{aligned}
& a_{\text {max }}(t)=G \frac{m}{D^{2}+r^{2}-2 D r} \\
& a_{\text {min }}(t)=G \frac{m}{D^{2}+r^{2}+2 D r}
\end{aligned}
$$

Half of the absolute change (amplitude) is

$$
\Delta a=\frac{a_{\max }(t)-a_{\min }(t)}{2}
$$

a)

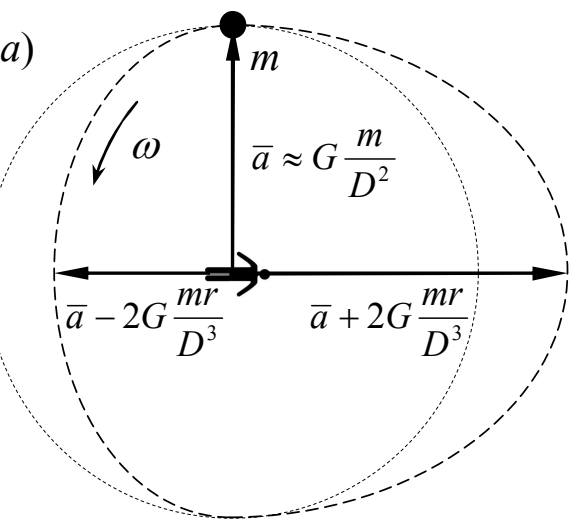

b)

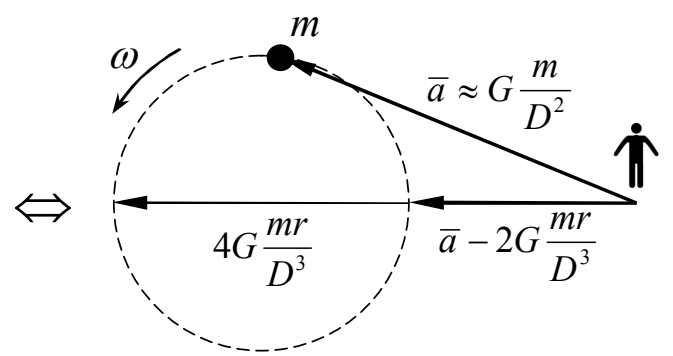

Fig. 12. Components of gravity field intensity $(D>>r): a)$ seen by rotating observer, $b$ ) caused by a single body rotating around a distant point.

Taking into account (56) and (57), the following are obtained

$$
\begin{gathered}
\Delta a=\frac{G}{2} \frac{m}{D^{2}+r^{2}-2 D r}-\frac{G}{2} \frac{m}{D^{2}+r^{2}+2 D r} \\
\Delta a=\frac{G}{2} m \frac{\left(D^{2}+r^{2}+2 D r\right)-\left(D^{2}+r^{2}-2 D r\right)}{\left(D^{2}+r^{2}\right)^{2}-4 D^{2} r^{2}} \\
\Delta a=\frac{G}{2} m \frac{4 D r}{D^{4}+2 D^{2} r^{2}+r^{4}-4 D^{2} r^{2}}=2 G m \frac{D r}{D^{4}-2 D^{2} r^{2}+r^{4}} \\
\Delta a=2 G \frac{D m r}{\left(D^{2}-r^{2}\right)^{2}}=2 G \frac{m r}{D^{3}} \frac{1}{\left(1-(r / D)^{2}\right)^{2}}>2 G \frac{m r}{D^{3}} \\
D>r \quad \Rightarrow \quad \Delta a \cong 2 G \frac{m r}{D^{3}} \sim \frac{m r}{D^{3}}
\end{gathered}
$$

In this case the amplitude of gravitational wave is inversely proportional to the third power of distance between the observer and source, i.e. $D^{3}$, Figure 12 . If $D>>r$, then different bodies will be the source of gravitational wave of a similar amplitude, if the value of $m r / D^{3}$ is similar for these bodies.

From both analyzed cases it appears that changes in gravitational field intensity from heavy objects located at large distances from the observer will be similar to changes in gravitational field intensity from light objects located at smaller distances from the observer. 
Gravitational waves in Newton's gravitation and criticism of gravitational waves resulting from the General Theory of Relativity (LIGO) - Roman Szostek, Paweł Góralski, Kamil Szostek

\section{LIGO experiment}

In 2016, the research team at the Laser Interferometer Gravitational-Wave Observatory (LIGO) [1] announced that it had indirectly measured the gravitational wave as predicted by GTR. Measurements were carried out simultaneously at two sites in Hanford and Livingston. Both measured signals were consistent with the course of gravitational wave resulting from GTR. The wave that was measured was to come from two black holes rotating around each other and falling on each other. The mass of black holes were estimated at 36 mass of the Sun $\left(\approx 7,2 \cdot 10^{31} \mathrm{~kg}\right)$ and 29 mass of the Sun $\left(\approx 5,8 \cdot 10^{31} \mathrm{~kg}\right)$. When they collided, they were distant of $410 \mathrm{Mpc}=1337260000$ light years $\left(\approx 1,27 \cdot 10^{25} \mathrm{~m}\right)$. Black holes falling down the spiral were, according to predictions of GTR, the source of gravitational wave understood as cyclical changes of the gravitational field intensity.

The LIGO experiment consists in searching for correlations between the signals measured at two Hanford and Livingston centers and the theoretical signal resulting from the GTR model. If these three signals are correlated, then it is concluded that a real signal was measured in the LIGO. Since the gravitational wave propagates at a finite speed of light, the two measured signals can be shifted in time.

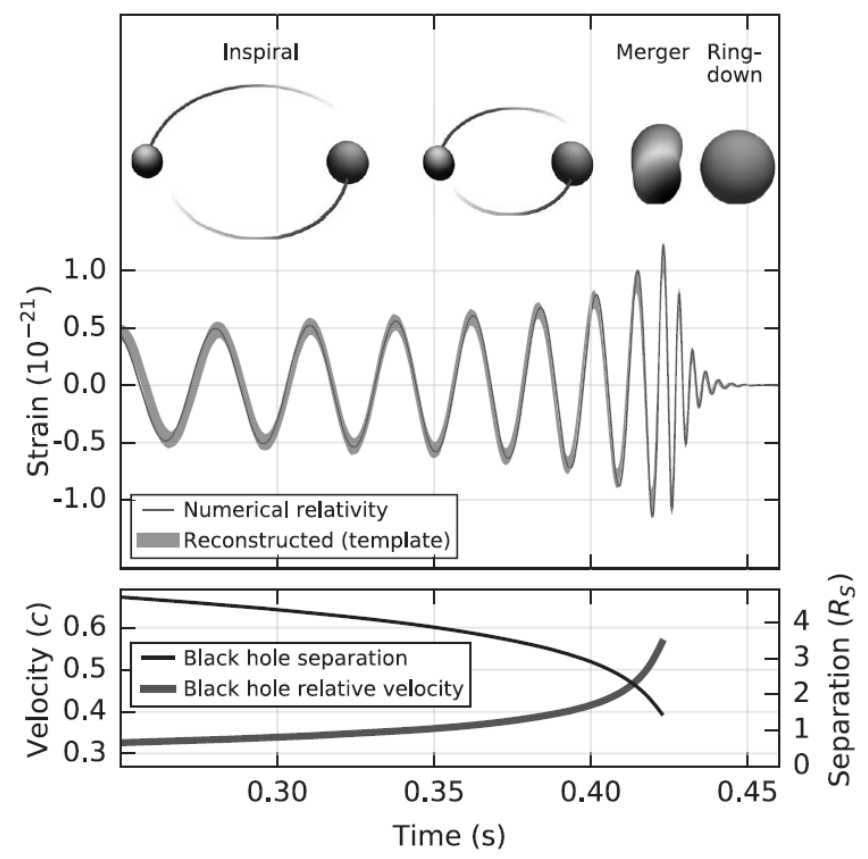

Fig. 13. Gravitational wave diagram given by the LIGO (GW150914). Top: amplitude resulting from the numerical model. Bottom: velocity of black holes and their distance given in the Schwarzschild's rays. Source [1] and [5].
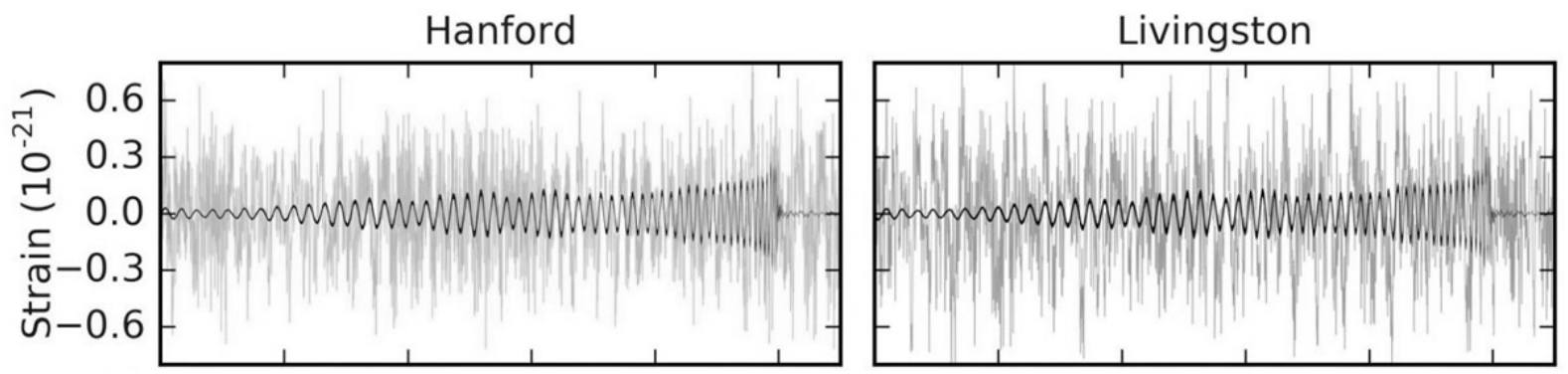

Fig. 14. The signal measured in LIGO is at the noise level (GW151226). The diagrams show the signal after filtering with the best fitted course resulting from the model. Source [2].

Figure 13 shows the first indirect measurement of gravitational wave announced by the LIGO (GW150914). This wave has amplitude, which grows in the last phase of bodies moving 
Gravitational waves in Newton's gravitation and criticism of gravitational waves resulting from the General Theory of Relativity (LIGO) - Roman Szostek, Paweł Góralski, Kamil Szostek

close each other. The figure shows that when black holes come close each other, the amplitude of the gravitational wave increases. The amplitude of gravitational wave disappears only after the collision of bodies.

Figure 14 shows the second measurement of gravitational wave announced by the LIGO (GW151226). As you can see in figure, the signals measured in the LIGO have values below the noise level. Searched gravitational wave had to be recovered with a use of filters made in the form of computer analysis. In this case, there is always a risk that the signal found is a result of used filters and not of actual measurement. In addition, in the noise it is possible to find the correlation with any pre-set signal. The study was conducted by Granger Clive, who discovered the phenomenon of coincidence in macroeconomic time series [4], for which he received the Nobel Prize in Economics in 2003. Therefore, if some stochastic signals were measured in Hanford and Livingston, it is almost certain that in a sufficiently long period of observation two very short signals will be observed, corresponding to searched signal. The correlation of such signals may be entirely incidental and not due to a common cause. With the LIGO, the risk is extremely high, as each collision of black holes is one-off. As a result of collision, the system of rotating black holes is destroyed and the measurement can never be repeated.

The above doubts are confirmed by the research published in paper [3]. The authors analyzed the noise available by the LIGO team. They detected that noise from both centers, although it should be independent, is often correlated. In addition, there is a correlation between the noise measured in Hanford and Livingston for the time shift between these noise levels, similar to that between the recorded signals that were supposed to come from black holes.

It is possible to verify whether the phenomenon of coincidence occurs during the measurement of gravitational wave. Check how often the required gravitational wave signal is present in only one interferometer. If the desired signal appears in one interferometer, but at the same time (with acceptable time shift) it does not appear in other, then it is not a gravitational wave measurement, but a random correlation (coincidence). Such situations will indicate that there are random correlations with the desired signal. If random correlations occur in one interferometer, they are of course less frequent in two interferometers at the same time. Such a situation is going to be interpreted incorrectly as a measurement of real gravitational wave. However, if correlations between measured noises with searched signal occur only simultaneously in both interferometers, then correlations are not incidental, but have a common cause. Since the LIGO interferometers conduct directional measurements (the wave from some directions can be measured and from others not), it would be beneficial to have two different interferometers parallel to each other in order to investigate whether there is a phenomenon of coincidence. Then each real wave would be measured in the same way by both interferometers. Unfortunately, the interferometers in Hanford and Livingston are built in such a way that their arms are inclined towards each other. In this case, one can measure the signal and the other cannot, if it is from the correct direction. Such an uneven setting of interferometers cause that it is difficult to demonstrate the compatibility of conducted measurements there with the model is incidental.

The course of gravitational wave presented by the LIGO team differs significantly from the course that results from the Newton's law of gravitation. The comparison refers to two rotating bodies. According to the LIGO, the amplitude of gravitational wave increases when bodies come close to each other (Figures 13 and 14). The amplitude of Newton's gravitational wave decreases in proportion to the bodies coming close to each other (Figure 4).

From the above it results that gravitational wave predicted by GTR has a different cause than the Newton's gravitational wave. The Newton wave is a result of variable, geometric layout of bodies in space. If the wave resulting from GTR had the same cause, it should follow a similar course. It is necessary to remembered that Newton's law of gravitation is one of the most experimentally verified physical laws. Of course, it was experimentally verified for bodies of relatively small mass. The LIGO measurements relate to bodies of much larger mass. If the course of gravitational wave is to be as published by the LIGO team, then for large circulating mass a 
Gravitational waves in Newton's gravitation and criticism of gravitational waves resulting from the General Theory of Relativity (LIGO) - Roman Szostek, Paweł Góralski, Kamil Szostek

completely new phenomenon must appear that does not appear in the Newton's law of gravitation. It is very interesting that such a new phenomenon has only been detected on the basis of GTR equations that have been proposed without any prior awareness of the existence of such a phenomenon.

Based on GTR, it was concluded that black holes lost a large part of their weight during the whole process of falling. Their mass was to radiate in a form of gravitational field [1]. After the collision, a black hole was created with a mass of about 63 mass of the Sun, i.e. 3 mass of the Sun less than the total mass of black holes colliding with each other. According to the Newton's law of gravitation, the gravitational force does not consume the body weight that generates it. This seems to be in line with the state of knowledge on the structure of matter. If the bodies lost weight due to generated gravity, then at least some atoms of matter would have to be disintegrated as a result of this process (or ageing consisting in reduction of their weight). Such a phenomenon is in no way investigated or confirmed under laboratory conditions.

According to simulations presented by the LIGO, the black holes that were the source of gravitational wave fell down on each other on a circular spiral in just a fraction of second. It is worth mentioning that such a way of their rotation is different from that which was observed directly in case of dual system PSR B1913+16 consisting of a pulsar and neutron star [6]. This system has been observed for several years. It was estimated that pulsar and neutron star, which rotate around each other at an average distance of about 2.65 rays of the Sun, would fall on each other after only 300 million years.

According to the LIGO, in both announced cases, black holes fell on each other in the spiral. In case of Newton's law of gravitation, two bodies related by gravity do not fall over each other in a spiral. Bodies could fall on each other in a spiral when they lose momentum for some reason, for example when they are moving in a dense medium. We have confirmed this with numerous simulations.

\section{Proposal for testing the LIGO Interferometer}

Every technical measuring device requires calibration or adjustment, because otherwise it is unknown what it measures. Neither calibration nor adjustment was applied in case of LIGO due to the lack of a controllable source of gravitational wave with known parameters that could be used as a standard. It will only be possible to check what the LIGO actually measures, if such a reference source of gravity wave is available.

The analysis presented in this article shows that it is possible to generate a model gravitational wave, which can be used for adjustment the LIGO. It is possible for that purpose to use e.g. body system as shown in Figure 1. The black holes in GW150914 formed this system before the collision. Figure 13 shows that when black holes had a velocity of $v=0.35 c$, they carried a full turn during $t=0.05 \mathrm{~s}$. On this basis it can be assumed that $r_{1} \approx 850 \mathrm{~km}$. On the basis of (54), taking into account equation (4), the following are obtained

$$
\begin{aligned}
& \frac{4 A_{x}^{\prime}}{9 G} \approx \frac{I\left(r_{1}+r_{2}\right)}{D^{4}}=\frac{r_{1} m_{1}\left(r_{1}+r_{1} \frac{m_{1}}{m_{2}}\right)}{D^{4}} \\
& \frac{4 A_{x}^{\prime}}{9 G} \approx \frac{850000 \cdot 7,2 \cdot 10^{31}\left(850000+850000 \frac{7,2 \cdot 10^{31}}{5,8 \cdot 10^{31}}\right)}{\left(1,27 \cdot 10^{25}\right)^{4}}=4,6 \cdot 10^{-57}
\end{aligned}
$$

According to (52), the average gravitational field intensity from black holes of GW150914 was as follows 
Gravitational waves in Newton's gravitation and criticism of gravitational waves resulting from the General Theory of Relativity (LIGO) - Roman Szostek, Paweł Góralski, Kamil Szostek

$$
\bar{a}_{x}^{\prime} \approx G \frac{m_{1}+m_{2}}{D^{2}}=6,67 \cdot 10^{-11} \frac{7,2 \cdot 10^{31}+5,8 \cdot 10^{31}}{\left(1,27 \cdot 10^{25}\right)^{2}}=5,4 \cdot 10^{-29}
$$

If for the system in Figure 1 we will accept that $m_{1}=10 \mathrm{~kg}, m_{2}=10 \mathrm{~kg}, r_{1}=10 \mathrm{~m}$, $D=10000 \mathrm{~km}$, then

$$
\frac{4 A_{x}^{\prime \prime}}{9 G} \approx \frac{I\left(r_{1}+r_{2}\right)}{D^{4}}=\frac{10 \cdot 10\left(10+10 \frac{10}{10}\right)}{\left(10 \cdot 10^{6}\right)^{4}}=2,0 \cdot 10^{-25}>>4,6 \cdot 10^{-57}
$$

According to (52), the average gravitational field intensity from considered light bodies is as follows

$$
\bar{a}_{x}^{\prime \prime} \approx G \frac{m_{1}+m_{2}}{D^{2}}=6,67 \cdot 10^{-11} \frac{10+10}{\left(10 \cdot 10^{6}\right)^{2}}=1,3 \cdot 10^{-23}>>5,4 \cdot 10^{-29}
$$

Based on the law (55) for the gravitational wave amplitude, or directly from (65) and (67), it results that the gravitational wave amplitude generated by two light bodies under consideration is more than $10^{30}$ orders of magnitude greater than the gravitational wave amplitude generated by black holes GW150914, when $r_{1}=850 \mathrm{~km}$. Even if there are some relativistic effects resulting from GTR that are not predicted by Newton's law of gravitation when the black holes come to each other, the difference in these amplitudes is so great that the LIGO system should be able to detect the wave from these light bodies.

The source of artificial gravitational wave shall be sufficiently distant from the LIGO for the wave reaching the LIGO to be virtually flat. The arms of LIGO interferometer are $4 \mathrm{~km}$ long, and therefore it seems that the distance of wave source by $D=10000 \mathrm{~km}$ should be sufficient. In order to avoid fraud, a completely different team of people should produce an artificial gravitational wave, accelerating the rotating bodies. The LIGO team shall be able to detect when these bodies are rotating.

The only technical problem in production of artificial gravitational waves is the rotation of bodies with sufficiently high frequency. According to the LIGO data, this system can only measure waves of a frequency greater than $40-150 \mathrm{~Hz}$ (in Figure 13 the gravity wave frequency is $40 \mathrm{~Hz}$ ). This limitation makes it impossible to rotate of any large bodies, but it is possible to rotate bodies of, let say, $1 \mathrm{~kg}$ on a radius of $r=1 \mathrm{~m}$.

\section{Conclusions}

Results announced by the LIGO team are presented as an experimental confirmation of the correctness of the General Theory of Relativity. However, in order for measurements taken by the LIGO to be unquestionable, it is necessary to make adjustments to this device. Only then will it be known whether the LIGO is able to detect gravitational waves and what it actually measures. For this purpose, it is possible to use circulating bodies, even of small mass, discussed in Chapter 6.

The existence of gravity waves results from the Newton's law of gravitation.

According to the Newton's law of gravitation, if the observer moves away from the source of gravity wave, then the gravitational field intensity decreases slower than the gravitational wave amplitude. If the source of wave is a system of two bodies related by gravity, as shown in Figure 1, then the amplitude decreases as $D^{4}$. If the source of wave is one body, as shown in Figure 8 , then the amplitude decreases as $D^{3}$.

Cyclical vibrations of the gravitational field intensity occur both in classical mechanics and in the General Theory of Relativity. However, they have different properties. The differences are as follows:

1. According to classical mechanics, when bodies come to each other, the gravitational field amplitude decreases (Figures 3 and 4). According to the General Theory of Relativity, when bodies come to each other, the amplitude of gravitational wave increases (Figures 13 and 14). 
Gravitational waves in Newton's gravitation and criticism of gravitational waves resulting from the General Theory of Relativity (LIGO) - Roman Szostek, Paweł Góralski, Kamil Szostek

This difference in the course of gravitational wave indicates that, according to GTR, the circulating large mass are accompanied by the phenomenon not occurring in Newton's law of gravitation, which is one of the best verified experimental physical laws. It is very interesting that such a new phenomenon has only been detected on the basis of GTR equations that have been proposed without any prior awareness of the existence of such a phenomenon.

2. In classical mechanics the gravitational interaction is not related to the loss of energy by the body that emits this interaction. According to the General Theory of Relativity, objects emitting gravitational waves lose their mass, carried in a form of gravitational waves [1].

3. According to classical mechanics, bodies cannot fall on each other in the spiral, if they do not lose momentum. They can fall on each other in a spiral, if they move in a dense medium and lose their momentum. Therefore, trajectories similar to the one shown in Figure 2 (wave in Figures 3 and 4) do not apply to bodies naturally related by gravity in an empty space. According to the results announced by the LIGO, as a part of GTR, bodies can fall on each other in the spiral, because they lose energy when they generate a gravitational wave.

4. Modulation of the gravitational field intensity causes changes in the dimension of bodies. Such a phenomenon is deformation of the shape of Earth during tides and outflows. According to GTR, such a phenomenon should be interpreted as a deformation of the space curve. According to classical mechanics, this is a simple deformation of the dimension of bodies caused by stress.

Measurements conducted in LIGO are on the noise level. This device is to measure relative changes of two lengths, four-kilometer arms at the level of one atom. Meanwhile the entire matter is filled up with various kinds of vibrations. The problem when measuring noise is the phenomenon of coincidence. It turns out that very often there are random correlations in rows of not connected with causality relations. Granger Clive's discovery of this phenomenon was honored with the Nobel Prize for Economics in 2003. The phenomenon of coincidence seriously undermines the credibility of measurements announced by the LIGO team.

It is possible to verify whether the phenomenon of coincidence occurs when measuring the gravitational wave (Chapter 5). You should check how often the searched signal, resulting from the model of GTR field, is present in only one interferometer. If it happens that such a signal occurs only in one interferometer, but at the same time (with acceptable time shift) it does not occur in other interferometer, then the detection of searched signal does not mean discovery of the gravitational wave. If interferometers actually measure the gravitational wave, then it should be recorded simultaneously by all the interferometers (taking into account their angles of inclination). The presence of a signal in only one interferometer will prove that the desired signals are present in measurements at random (random noise) or have a local cause.

According to the General Theory of Relativity, mass do not interact gravitational with each other by the gravity force, but because they bend the space. If, according to the General Theory of Relativity, gravity manifests itself as a curvature of space, then it is difficult to explain tides and outflows. At tides and outflows, the differences in water levels reach several meters. It follows that the changes in curvature of space, postulated by GTR, caused by variable gravitation of the Moon are very large. The problem is why tides and outflows are very clear, while the deformations of mainland are invisible. If the mass of Moon bends the space, it is the same when that space is filled with water and when it is filled with the mainland. Therefore, sea water should be deformed in the same way as the mainland. The banks should rise in the same way as the water. Then, of course, tides and outflows would be invisible. However, because they are visible, it follows that the mass of Moon does not distort space, but rather the matter. The water is malleable, and therefore it deforms more than stiff rocks. This proves that the assumption of GTR, that gravity is a curvature of space, is incorrect.

Presented analysis shows that "gravitational wave", calculated on the basis of GTR, is not in fact a wave understood as a carrier of gravity. This is a normal modulation (i.e. cyclic 
Gravitational waves in Newton's gravitation and criticism of gravitational waves resulting from the General Theory of Relativity (LIGO) - Roman Szostek, Paweł Góralski, Kamil Szostek

change) of the gravitational field intensity caused by the motion of two rotating bodies. This is evidenced by the fact that frequency of the "gravitational wave" resulting from GTR is closely related to the frequency of rotating bodies (for two bodies the frequency of "gravitational wave" is twice as high as the frequency of rotating bodies). In this article we showed that according to the Newton's law of gravitation, rotating bodies also causes modulation of gravity field intensity with a frequency two times the frequency of spinning of bodies (i.e., the same way as in the framework of GTR).

If two light sources (e.g. light bulbs as shown in Figure 15) rotate, they shall also produce modulated intensity lighting. In this case also the frequency of modulation of the light intensity will be closely related to the frequency of rotating bulbs. This modulation of light intensity has nothing to do with the photon's properties. It has nothing to do with the frequency (its color) of light produced by these light bulbs. The frequency that can be assigned to photons (i.e. frequency of light produced by the bulbs) is something completely different and independent of the frequency of modulation of the light intensity, caused by rotating bulbs.

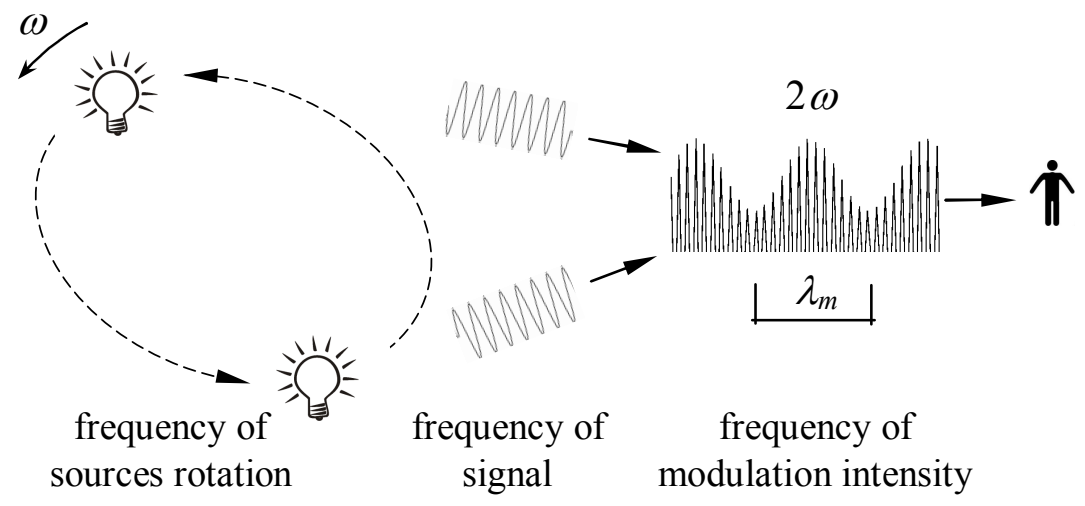

Fig. 15. Frequency of light intensity modulation is something else than the frequency of light signal.

It is the same as in case of the gravitational field intensity modulated by rotating bodies. Modulation of gravitational field intensity has no connection with the properties of hypothetical graviton. Because the model of field on which GTR is based is mathematically complex, the normal modulation of gravitational field intensity, caused by rotating bodies, was wrongly interpreted as a gravitational wave, understood as a carrier of gravity [1], [2], [5].

In this article we called the normal modulation of gravitational field intensity as a gravitational wave, because this was done in case of "gravitational waves" resulting from GTR.

\section{Bibliography}

[1] Abbott B. P. et al., Observation of Gravitational Waves from a Binary Black Hole Merger, Physical Review Letters, Vol. 116, Iss. 6, 061102, 1-16, 2016.

[2] Abbott B. P. et al., GW151226: Observation of Gravitational Waves from a 22-Solar-Mass Binary Black Hole Coalescence, Physical Review Letters, Vol. 116, Iss. 24, 241103, 1-14, 2016.

[3] Creswell James, Hausegger Sebastian, Jackson Andrew D., Liu Hao, Naselsky Pavel, On the time lags of the LIGO signals, arXiv 2017, 1-28, https://arxiv.org/abs/1706.04191.

[4] Granger Clive W. J., Some Properties of Time Series Data and Their Use in Econometric Model Specification, Journal of Econometrics, Vol. 16, 121-130, 1981. 
Gravitational waves in Newton's gravitation and criticism of gravitational waves resulting from the General Theory of Relativity (LIGO) - Roman Szostek, Paweł Góralski, Kamil Szostek

[5] Royal Swedish Academy of Sciences. The laser interferometer gravitational-wave observatory and the first direct observation of gravitational waves, Stockholm, Scientific Background on the Nobel Prize in Physics, 1-18, 2017.

[6] Taylor J. H., Weisberg J. M., A new test of general relativity - Gravitational radiation and the binary pulsar PSR 1913+16, Astrophysical Journal 253, 908-920, 1982.

[7] Hartle James B., Gravity. An Introduction to Einstein's General Relativity, published by Pearson Education, publishing as Benjamin Cummings, 2003. 